\title{
STUDY OF ADENOSINE DEAMINASE (ADA) ACTIVITY IN CEREBROSPINAL FLUID (CSF) IN TUBERCULOUS MENINGITIS IN CHILDREN.
}

\author{
Biochemistry \\ Dr. Md Raza Sami Junior Resident, Department Of Biochemistry, PMCH, Patna. \\ Dr. Alok Kumar* Junior Resident, Department Of Biochemistry, PMCH, Patna. *Corresponding Author \\ Dr. Farhan Usmani Associate Professor, Department Of Biochemistry, PMCH, Patna

\section{Dr. Ram Binay} \\ Sinha \\ Professor, Department Of Biochemistry, PMCH, Patna.
}

\begin{abstract}
BACKGROUND: Among all other causes of meningitis Tuberculous meningitis (TBM) is an endemic disease in developing countries with an incidence of 7 to $21 \%$. But the available methods of diagnosis of TBM have too low sensitivity and specificity. In these situations, Adenosine Deaminase (ADA) estimation has been found to be profoundly useful as it is less time consuming, cost effective and affordable.

AIMS AND OBJECTIVE: To evaluate the diagnostic significance of cerebrospinal fluid (CSF) adenosine deaminase (ADA) activity in tubercular meningitis (TBM) in children.

MATERIALS AND METHODS: In Our Study, 200 consecutive medical ward in-patients of Pediatrics department Patna medical college, Patna who had features of meningitis were prospectively enrolled and included in the study after informed consent.

RESULTS: Out of 200 patients 112 were males and 88 were females. Cases of tuberculous meningitis constituted $34 \%$ of the study group and bacterial and viral meningitis were $22 \%$ and $23 \%$ respectively. while $21 \%$ patients from study group had no any type of meningitis. CSF ADA cutoff of $10 \mathrm{U} / \mathrm{L}$ considered in our study has $100 \%$ sensitive and $97.06 \%$ specific.

CONCLUSION: detection of elevated level of ADA in CSF in suspected cases of meningitis in children will be a guide in the diagnosis of TBM so that early treatment will be started and will lead to improved outcome.
\end{abstract}

\section{KEYWORDS}

\section{Adenosine Deaminase, Tuberculous Meningitis.}

\section{INTRODUCTION:}

Meningitis is inflammation of the meninges (Pia, Arachnoid and Dura mater) covering the brain and spinal cord. The most common cause of meningitis is infections due to viruses, bacteria, mycobacterium, fungi and other microorganisms. The non-infectious causes include malignancy, chemical compounds, drugs and inflammatory conditions like CNS sarcoidosis. SLE, Behcet's syndrome etc. Meningitis can also be classified according to the temporal profile as acute, sub acute and chronic types. [1]

Meningitis is a medical emergency as it can be life threatening because of the inflammation proximity to brain and spinal cord. If not recognized earlier, it can lead to serious long term neurological sequelae like deafness, epilepsy, hydrocephalus and cognitive deficits. [2]

Lumbar puncture and CSF analysis are used to diagnose or exclude meningitis. Management involves prompt administration of antibiotics, and in appropriate situations antiviral and anti tuberculous agents according to the organism suspected. Steroids are used as adjunctive agent to prevent the complications from overactive inflammation. [3]

Among all other causes of meningitis tuberculous meningitis (TBM) is an endemic disease in developing countries with an incidence of 7 to $21 \%$ according to Gupta B K et al (2010). It presents with gradual onset of symptoms and results in irreversible neurological complications and death if there is delay in the diagnosis and start of effective treatment. But the available methods of diagnosis of TBM have too low sensitivity and specificity. Detection of acid fast bacilli (AFB) by light microscopy of the CSF smear is rapid and specific method, with a detection rate of only 30 $40 \%$ according to Steingart K R et al (2006). Sensitivity of mycobacterium culture in Lowenstein- Jensen (L-J) medium is higher than microscopy but it needs several weeks of incubation. A number of genotypic assays based on nucleic acid amplification have been designed according to $\mathrm{Abe} \mathrm{C}$ et al. However, high costs involved in tests preclude their use especially in developing countries. [4]

In these situations, Adenosine Deaminase (ADA) estimation has been found to be profoundly useful as it is less time consuming, cost effective and affordable. ADA is an enzyme in the salvage pathway which is found in abundance in active T-lymphocytes. It is released by the $T$ cells during cell mediated immune response to the tubercle bac illi. ADA levels have been considered by several researchers to differentiate tuberculous and non-tuberculous diseases. Its role in differentiating the tuberculous pleural, pericardial effusion and ascites from other Causes has been well established. Several studies have also emphasized its importance as a simple, rapid, cost effective and fairly specific test in distinguishing tuberculous meningitis from other causes of meningitis. Hence an attempt was made to estimate the CSF ADA level in patients with suspected cases of meningitis and throw light on its use in differentiating the various types of meningitis and early diagnosis of tuberculous meningitis. [5]

\section{MATERIALS AND METHODS:}

This is a cross sectional study conducted in the department of Bioch e mistry, Patna medical college, Patna. The diagnosis of meningitis was made on the basis of clinical symptoms and signs.

\section{INCLUSION CRITERIA:}

Children of age group 6 months to 12 years with the features suggestive of meningitis were included in this study.

\section{EXCLUSION CRITERIA:}

Children of age less than 6 months and age more than 12 years were excluded from the study.

The defferent types of meningitis patients were separated on the basis of CSF cytochemistry and clinical features. Tuberculous meningitis was conformed if CSF culture yielded $\mathrm{M}$. tuberculosis or a positive Ziehl - Neelson staining. Probable disease was diagnosed in the presence of a lymphocytic pleocytosis in the CSF with high protein content and low glucose content, negative bacterial and fungal cultures. Acute bacterial meningitis was diagnosed in the patients with CSF neutrophilia, high protein content, low glucose content and a positive gram staining and bacterial culture.Viral meningitis was diagnosed if there was predominantly pleocytosis in the CSF with a normal or mildly raised protein content, a normal glucose content and negative bacterial, fungal and mycobacterial cultures

History, clinical examination and laboratory investigation were carried out in all patients and details recorded in a predesigned performa. Important clinical details regarding duration of fever, signs of meningeal irritation, focal neurological deficits and cranial nerve palsies were elicited. Laboratory examination including CSF examination (appearance, cell counts, biochemistry, Gram staining, 
Acid fast bacilli and India ink stain), blood counts, blood culture and sensitivity, Mantoux test, HIV test. Based on clinical and laboratory data patient's type of meningitis was confirmed and treatment started accordingly.

CSF Adenosine deaminase activity was measured at 37 degree Celsius according to the method of Guisti and Galanti based on Berthelot reaction that is the formation of coloured indophenol complex and ammonia librated from adenosine and quantified spectropho to metrically. One unit of ADA is defined as the amount of enzyme required to release $1 \mathrm{mmol}$ of ammonia/min from adenosine at standard assay conditions. Results were expressed as units per liter per minute (U/L/min). Slectra Pro M fully auto analyzer and Adenosine deaminase (ADA) Activity Assay kit (Colorimetric) (ab204695) were used for the estimation of ADA activ ity in CSF.

\section{STATISTICALANALYSIS:}

Results obtained were arranged in tables and graphs and it was analysed statistically. Sigma Stat statistical software was used in the study.

\section{RESULTS:}

The present study shows that the percentage of TBM is more in age group of $1-10$ years. While controls are almost uniformly distributed in all age group i.e. 6 months to 12 years[Table 1]. Out of 68 cases, 34 were males and 34 were female i.e; Tuberculous Meningitis found equally frequent in both sexes. While out of 132 controls, 78 were male i.e; more frequent in males. CSF ADA level was highest in Tuberculous Meningitis[Table2]. The mean value being 15.8647 U/L with a standard deviation of 2.8295 . The mean value of ADA in control groups was $4.6678 \mathrm{U} / \mathrm{L}$ with SD of 1.7246. [Table 3 ]

Out of the 200 patients, 136 had ADA $<10 \mathrm{U} / \mathrm{L}$ out of this 132 belonged to control group i.e. Non Tuberculous group and rest of had Tuberculous Meningitis. About 64 patients had CSF ADA $\geq 10 \mathrm{U} / \mathrm{L}$ and all of them belonged to Tuberculous Meningitis group.[Table 4]

Table - 1 Distribution of age among cases \& Controls

\begin{tabular}{|c|c|c|c|c|}
\hline \multirow{2}{*}{ Age in Yrs } & \multicolumn{2}{|c|}{ Cases } & \multicolumn{2}{c|}{ Controls } \\
\cline { 2 - 5 } & Numbers & Percentage & Numbers & Percentage \\
\hline$<1$ & 2 & $3 \%$ & 10 & $8 \%$ \\
\hline $1-5$ & 36 & $53 \%$ & 50 & $38 \%$ \\
\hline $5-10$ & 22 & $32 \%$ & 48 & $36 \%$ \\
\hline $10-12$ & 8 & $12 \%$ & 24 & $18 \%$ \\
\hline Total & 68 & $100 \%$ & 132 & $100 \%$ \\
\hline Range & $0.5-12$ & & $0.5-12$ & \\
\hline Mean & 4.854 & & 5.5888 & \\
\hline SD & 3.1309 & & 3.4712 & \\
\hline
\end{tabular}

Table - 2 Mean CSF ADA among Cases and Controls

\begin{tabular}{|c|c|c|c|}
\hline & Numbers & $\begin{array}{c}\text { Mean CSF ADA } \\
\text { (in U/L) }\end{array}$ & $\begin{array}{c}\text { Standard Deviation } \\
\text { (in U/L) }\end{array}$ \\
\hline Cases & 68 & 15.8647 & 2.8295 \\
\hline Control & 132 & 4.6678 & 1.7246 \\
\hline Total & 200 & & \\
\hline
\end{tabular}

Table - 3 Distribution of Cases (TBM) and Controls with respect to CSF ADA cut off value $-10 \mathrm{u} / \mathrm{L}$

\begin{tabular}{|c|c|c|}
\hline CSF ADA Level (U/L) & Cases & Controls \\
\hline$<10$ & 4 & 132 \\
\hline$\geq 10$ & 64 & - \\
\hline Total & 68 & 132 \\
\hline
\end{tabular}

Table - 4 Diagnostic Performance of CSF ADA(at 10 U/L cut off) in relation to type of Meningitis

\begin{tabular}{|c|c|c|c|}
\hline Disease & Test (CSF ADA) & & Total \\
\hline & $\geq 10$ & $<10$ & \\
\hline TBM & $64(\mathrm{a})$ & $4(\mathrm{c})$ & $(\mathrm{a}+\mathrm{b}) 68$ \\
\hline Non TBM & $0(\mathrm{~b})$ & $132(\mathrm{~d})$ & $(\mathrm{b}+\mathrm{d}) 132$ \\
\hline Total & $(\mathrm{a}+\mathrm{b}) 64$ & $136(\mathrm{c}+\mathrm{d})$ & $200(\mathrm{a}+\mathrm{b}+\mathrm{c}+\mathrm{d})$ \\
\hline
\end{tabular}

\section{DISCUSSION:}

In the present study, Tuberculous meningitis(TBM) group included 68 cases with 34 males \& 34 females, while the control group included
132 patients with 78 males \& 54 females. Both groups were similar in their distribution of age ( 6 months to $12 \mathrm{yrs}$ ). In this study ADA activity in CSF was evaluated.

There is considerable urgency in establishing the early and correct diagnosis in patients with meningitis since specific therapy is most effective when instituted early in the course of illness.

The diagnosis of TBM is difficult task throughout the world. Delayed diagnosis and treatment is a regular feature, so there is urgent need to improve the diagnostic tests. Detection of Acid fast bacilli (AFB) in CSF is best diagnostic test for TBM but it has detection rate only 15 to $20 \%$. It takes several weeks (4-8 weeks) to obtained positive culture results in conventional Lowenstein-Jensen(L-J) medium and positive in $50 \%$ of cases only. Culture remain the gold standard to make the diagnosis of TBM. Contemporary systems such as radiometric BACTEC and biphasic culture (Roche SepticChek) improve the speed of mycobacterium recovery in 7 to 10 days. Therefore waiting for these methods or culture reports to confirm the diagnosis are time consuming. Delayed treatment will lead to an irreversible brain damage and other complications. Many tests have been developed to diagnose TBM but best diagnostic test still remain controversial except detection of mycobacterium. Clinical features, CSF cytology, biochemistry and microbiology results are many a time inconclusive with regard to confirmation of diagnosis. So in suspected meningitis patients, specific treatment for tuberculous and non tuberculous( bacterial or viral) etiology is usually begun on the basis of presumptive clinical diagnosis.

Moreover in the diagnostic evaluation of meningitis, sophisticated methods are necessary which are not routinely available in institutions in the developing countries. In this situation, CSF Adenosine deaminase (ADA) estimation which is simple, cost effective and rapid, helps in differentiating the etiology of meningitis when other finding are ambiguous. Hence this simple test aids in prompt and appropriate diagnosis and early treatment of this tuberculous meningitis and helps in saving many lives.

In the present study it is clear that Adenosine deaminase level was clearly higher in patients with tuberculous meningitis in our study, consistent with various ealier studies, like Satya Vati Rana et al (2004), Sharad Jain et al (2005), Amulya C Belagavi et al (2008) and Anil Chandra et al (2012) etc.[6]

By using $10 \mathrm{U} / \mathrm{L}$ as the cut off value, the test had sensitivity of $100 \%$ and specificity $97.06 \%$ in identifying cases of tuberculous meningitis. The positive predictive value and negative predictive value were $94.12 \%$ and $100 \%$ respectively. The positive likelihood and negative likelihood ratio 34 and 00 respectively. The higher level of ADA in tuberculous meningitis was statistically significant $(\mathrm{p}<0.001)$.

In present study the Case group (TBM) have mean value of ADA in CSF was $15.8647 \mathrm{U} / \mathrm{L}$ with a standard deviation of $2.8295 \mathrm{U} / \mathrm{L}$. Present study is considerably close to that reported by other workers( 11.7-15.7 U/L) for eg: Anil Chandra and et al(2010), Dr Aniruddha Debnath et al(2015) etc. The range of CSF ADA level in TBM in our study was $1.7 \mathrm{U} / 1$ to $23.3 \mathrm{U} / 1$. The mean ADA level in CSF in control group was $4.6678 \mathrm{U} / 1$ with a standard deviation of 1.7246 . This shows the higher level of CSF ADA in TBM. It was also observed that out of 68 cases of TBM 64 cases had ADA level in $\mathrm{CSF} \geq 10 \mathrm{U} / \mathrm{L}$. [7]

In present study ADA level in CSF is elevated in TBM significantly( $\mathrm{p}<$ 0.001). Similar results were reported by Amulya C Belagavi et al (2008) with mean value of ADA in CSF was $14.14 \pm 7.44 \mathrm{U} / \mathrm{L}$ in TBM group. Present study is also in confound with study of Gupta et al (2010) who in their study established that CSF ADA level of $10 \mathrm{U} / \mathrm{L}$ as a cut off value exhibited $94.73 \%$ sensitivity and $90.47 \%$ specificity in differentiating tuberculous from non tuberculous meningitis. Various other studies used different cut off level for ADA as 3.3 U/L in the study of Rajendra Prasad et al (1991) with sensitivity of $100 \%$ and specificity of $97.87 \%, 6.5 \mathrm{U} / \mathrm{L}$ in the study of Rajesh Baheti et al with sensitivity of $95.83 \%$ and specificity of $92.85 \%$ respectively.[8]

CSF ADA level remain elevated in tuberculous meningitis whereas $\mathrm{C}$ Reactive protein(CRP) level in CSF is elevated in pyogenic meningitis. Level of ADA and CRP in CSF are low in viral meningitis. Therefore measurement level of ADA along with CRP in CSF can differentiate among tuberculous, bacterial and viral meningitis. [9] 
So detection of elevated level of ADA in CSF in suspected cases of meningitis in children will be a guide in the diagnosis of TBM so that early treatment will be started and will lead to improved outcome.[10]

\section{CONCLUSION:}

The conclusions that can be drawn from our study, regarding CSF ADA is a simple, time saving, inexpensive indirect test that help in identifying the type of meningitis and differentiating tuberculous from nontuberculous etiology. Further among non tuberclous group of meningitis, CSF ADA values are lowest in viral meningitis and thus it can aid in distinguishing bacterial from viral etiology. CSF ADA estimation can be done at routine labs spectrophotometrically and assayed according to the method of Guisti.CSF ADA cut-off of $10 \mathrm{U} / \mathrm{L}$ considered in our study has $100 \%$ sensitive and $97.06 \%$ specific in differentiating tuberculous from nontuberculous etiology as early as possible, facilitating immediate management of these patients preventing morbidity and mortality due to delay in management.

However as the study was limited to small population, further study and analysis of a larger group would definitely give an insight into the further finer relationship between Adenosine deaminase activity in CSF and clinical severity and outcome of the patients suffering from tuberculous meningitis.

\section{REFERENCES:}

AAdams and R.A. Harkness. A Brief Communication: ADA activity in thymus and other human tissues. Clin. Exp. Immunol 1976; 26: 647-649

2. Abe C, Hirano K, Wada M, Kazumi Y, Takahashi M, Fukasawa Y, Yoshimura T, et al. Detection of Mycobacterium tuberculosis in clinical specimens by polymerase chain reaction and Gene probe Amplified Mycobacterium Tuberculosis Direct Test. J Clin Microbiol 1993;31 (12): 3270-3274

3. Allan H. Rooper, Martin A Samuels. Adams and Victor's Principles of Neurology. 9th ed. The McGraw-Hill Companies, Inc. 2009;32:668-690 Infections of the Nervous System and Sarcoidosis and 2009;33:712-713 Viral Infections of the Nervous System, Chronic and Sarcoidosis and 2009;33:7

4. Amulya C Belagavi, M. Shalini. Cerebrospinal Fluid C Reactive Protein and Adenosine Demanise in Meningitis in Adults. JAPI; 2011;59: 557 - 560

5. Anil Chander, Chandrika D. Shrestha . CSF ADA level as a diagnostic marker in TBM in adult Nepalese patients. Asian Pac J Trop Dis2013; 3(1): 16-19

6. Dr. Aniruddha Debnath, Dr. Sunita singh, Dr. Momota Naiding, Dr. R.N. Chaubey. Diagnosis of Meningitis with special Referance to Adenosine Deminase and C-Reactive Protein Level In CSF. IOSR - JDMS; e-ISSN 2017;16(6): 2279 - 0853, P-ISSN Protein Level In CSF. IOSR - JDMS;

7. G Thwaites, Hein TT, NTH Mai, F Drobnicwiki, K MCAdam, Ja Farrar. Tuberculous Meningitis; Neurological aspect of Tropical Disease. J. Neurol Neurosurg Psychiatry 2000; 68: 289-299

8. Guisti G, Galanti B; Adenosine deaminase: colorimetric method. In Methods of Enzymatic Analysis, 5th edition. Edited by: Bergmeyer HU. Weinheim (Germany): Verlag Chemie, 1984;5:315-323

9. T. Hima Bindu, R. Maheshwara Reddy. Role of cerebrospinal Fluid ADA activity in the diagnosis of TBM in children IJCP; 2019; 4 (2):411-414

10. William W Campbell. Dejong's The Neurological Examination. 6th ed. India: Jaypee Brothers Medical Publisher; 2020;6(52):617-618; 